\title{
Involvement of genes encoding ABI1 protein phosphatases in the response of Brassica napus $L$. to drought stress
}

\author{
Danuta Babula-Skowrońska ${ }^{1}$ - Agnieszka Ludwików ${ }^{2}$ - Agata Cieśla $^{2} \cdot$ Anna Olejnik $^{3}$ • \\ Teresa Cegielska-Taras ${ }^{3}$ Iwona Bartkowiak-Broda ${ }^{3} \cdot$ Jan Sadowski $^{2}$
}

Received: 6 February 2015 / Accepted: 22 May 2015 / Published online: 10 June 2015

(C) The Author(s) 2015. This article is published with open access at Springerlink.com

\begin{abstract}
In this report we characterized the Arabidopsis ABII gene orthologue and Brassica napus gene paralogues encoding protein phosphatase $2 \mathrm{C}$ ( $\mathrm{PP} 2 \mathrm{C}$, group $\mathrm{A})$, which is known to be a negative regulator of the ABA signaling pathway. Six homologous $B$. napus sequences were identified and characterized as putative PP2C group A members. To gain insight into the conservation of $A B I I$ function in Brassicaceae, and understand better its regulatory effects in the drought stress response, we generated transgenic $B$. napus plants overexpressing $A$. thaliana ABII. Transgenic plants subjected to drought showed a decrease in relative water content, photosynthetic pigments content and expression level of $R A B 18$ - and RD19A-drought-responsive marker genes relative to WT plants. We present the characterization of the drought response of $B$. napus with the participation of $A B I 1$-like paralogues. The expression pattern
\end{abstract}

Danuta Babula-Skowrońska and Agnieszka Ludwików have contributed equally to this article.

Electronic supplementary material The online version of this article (doi:10.1007/s11103-015-0334-x) contains supplementary material, which is available to authorized users.

Danuta Babula-Skowrońska

dbab@igr.poznan.pl

$\triangle$ Jan Sadowski

jsad@amu.edu.pl

1 Institute of Plant Genetics, Polish Academy of Sciences, Strzeszyńska 34, 60-479 Poznan, Poland

2 Department of Biotechnology, Institute of Molecular Biology and Biotechnology, Faculty of Biology, Adam Mickiewicz University, Umultowska 89, 61-614 Poznan, Poland

3 Plant Breeding and Acclimatization Institute - National Research Institute, Research Division in Poznań, Strzeszyńska 36, 60-479 Poznan, Poland of two evolutionarily distant paralogues, BnaA01.ABI1.a and BnaC07.ABII.b in B. napus and their promoter activity in $A$. thaliana showed differences in the induction of the paralogues under dehydration stress. Comparative sequence analysis of both BnaABII promoters showed variation in positions of $c i$-acting elements that are especially important for ABA- and stress-inducible expression. Together, these data reveal that subfunctionalization following gene duplication may be important in the maintenance and functional divergence of the BnaABII paralogues. Our results provide a framework for a better understanding of (1) the role of ABI1 as a hub protein regulator of the drought response, and (2) the differential involvement of the duplicated BnaABII genes in the response of B. napus to dehydration-related stresses.

Keywords Brassica napus - Drought response - ABI1 protein phosphatase $\cdot$ Gene overexpression $\cdot$ Promoter activity

\section{Introduction}

Drought stress induces diverse responses at the cellular and molecular levels including the production of plant phytohormones, especially abscisic acid (ABA) (Swamy and Smith 1999; Zhu 2003). The core ABA signaling pathway consists of three important elements: ABA receptors (PYR/PYL/RCAR proteins), protein phosphatases type 2C (PP2Cs) and SNF1-related protein kinases (SnRK2s) (Yoshida et al. 2006; Ma et al. 2009; Fujita et al. 2009, Umezawa et al. 2010). In Arabidopsis, there are nine group A PP2Cs: ABI1, ABI2, HAB1, HAB2, HAI1, HAI2, HAI3, AHG1 and AHG3 (Gosti et al. 1999; Merlot et al. 2001; Yoshida et al. 2006; Rubio et al. 2009). It is widely 
accepted that group A PP2Cs have a key role in the ABA signaling pathway (Sheen 1998; Schweighofer et al. 2004; Ludwików 2015 for review). ABA stimulates plant responses to abiotic stresses such as drought, high salt and cold, and several research teams have shown that group A PP2Cs, including ABI1 (ABA Insensitive 1; Leung et al. 1994), are negative regulators of ABA signaling (Gosti et al. 1999; Merlot et al. 2001; Saez et al. 2004). Although it is known that PP2Cs interact with various targets to form a regulatory core (Ludwików 2015 for review) that modulates cellular processes and stress response pathways (Nemhauser et al. 2006; Ludwików et al. 2009; Umezawa et al. 2009; Geiger et al. 2010; Ludwików et al. 2014), their role in the acclimation of plants to drought stress remains unclear. Hence it is important to investigate further the function of ABI1 and other group A PP2Cs in the stress responses of plants, including economically important crops with complex genomes typically carrying multiple gene homologues.

Although group A PP2Cs have been extensively analyzed in Arabidopsis, their orthologues (ancestrally related genes maintained in different species after speciation events) have also been identified and partially characterized in other plant species (Gonzalez-Garcia et al. 2003; Xue et al. 2008; Tougane et al. 2010; Ludwików et al. 2013; Yuan et al. 2013, Jia et al. 2013). In Brassica species, protein-encoding genes are generally triplicated relative to their counterparts in A. thaliana due to wholegenome duplication events (Babula et al. 2003; Parkin et al. 2005; Lysak et al. 2005; Babula et al. 2006; Ziolkowski et al. 2006; Wang et al. 2011) between 7.9 and 14.6 Mya. Despite the high degree of sequence similarity of their transcripts and the corresponding proteins, such paralogous genes often exhibit significant differences in expression patterns. This can result in increased overall gene expression levels and can provide a source of novel gene variants (Udall and Wendel 2006; Whittle and Krochko 2009). The potential for functional divergence among duplicated genes and consequent pleiotropic effects on stress responses, especially to stresses such as drought, is poorly described for crop plants including the Brassica species.

Currently, there is a paucity of information on the role of paralogous $A B I I$ copies (they are considered in-paralogues, i.e. they result from duplication after the last speciation event; Studer and Robinson-Rechavi 2009) in the regulation of the ABA signaling pathway in the Brassica species. Previous studies have focused on individual $A B I I$ gene copies in particular species, and have neglected to investigate paralogous examples (Yuan et al. 2013; Zhang et al. 2014).

In this study we have investigated the conservation of the role of the ABIl gene orthologue in Brassicaceae (Brassica napus vs. Arabidopsis) and the duplicated $A B I 1$ genes in $B$. napus with particular reference to the response to drought stress. We generated transgenic $B$. napus plants overexpressing the A. thaliana ABII orthologue to study corresponding changes in the drought-stress response, as well as the cumulative effect of all BnaABII-like genes under drought conditions. Overexpression of AtABII resulted in water loss and changes in chlorophyll accumulation indicating that these stress-related physiological responses are controlled by the ABI1-dependent ABA-signaling pathway. Transcript levels of known drought stress marker genes were also downregulated in transgenic plants compared to wild type B. napus. We also observed changes in the transcript levels of the endogenous $A B I 1$ homologues (BnaABII s) in wild type (WT) and transgenic B. napus to evaluate their involvement in the drought response. This aspect presents important insight into poorly understood dehydration-induced co-response of duplicated gene homologues occurring typically in plants. To determine whether the duplicated BnaABII genes are functionally redundant or whether they have evolved novel subfunctions, we performed a detailed analysis of two evolutionarily distant BnaABI1 paralogues, BnaA01.ABI1.a and BnaC07.ABII.b, and found evidence for structural and functional diversification. We demonstrated that the promoter activity of these paralogues in A. thaliana was sufficient to control tissuespecific expression, but resulted in different stress-induced expression. The combined results shed new light on diverse roles of the $A B I I$ gene family in the drought response plasticity in the Brassica polyploid. In-depth knowledge about the functional divergence of BnaABII members will facilitate future work on genetic engineering of Brassica species.

\section{Materials and methods}

\section{Plant material, growth and stress conditions}

Arabidopsis thaliana (L.) Heynh. ecotype Columbia (Col-0) and B. napus microspore-derived embryos of cv. Monolit (HR Strzelce, Poland) were used to generate homozygous transgenic plants. Plant growth conditions, exogenous $\mathrm{ABA}$ and drought stress treatments were described previously by Ludwików et al. (2013). The $B$. napus seeds were germinated in sterilized petri dishes on sand for 3 days at $25{ }^{\circ} \mathrm{C}$. After incubation, good quality seedlings with fully expanded green cotyledon were grown in pots $(7 \mathrm{~cm} \times 7 \mathrm{~cm} \times$ high $8 \mathrm{~cm} ; 2$ seedlings per pot; 20 pots per condition) filled with soil in a growth room under long-day conditions (16/8 h of light/ dark with $18{ }^{\circ} \mathrm{C}$ ). They were watered every 2 days for 3 weeks and fertilized weekly with Florovit $(0.5 \mathrm{ml} / \mathrm{L})$. The drought stress was induced in well-watered threeweek-old plants by withholding water for 5 days. These plants were then re-watered for 5 days after which leaves 
were collected. In parallel, control plants received water regularly. Relative water content (RWC) was calculated according to the formula: [(fresh weight - dry weight)/ (turgid weight - dry weight)] $\times 100$. For wounding treatment all rosette leaves were crushed with forceps, which effectively wounded $40 \%$ of the leaf area (Reymond et al. 2000). All measurements were made on three or more replicates.

\section{Identification and cloning of $A B I 1$ paralogues from the B. napus genome}

The A. thaliana ABII cDNA sequence (At4g26080) was used as a reference sequence to detect the corresponding $B$. napus EST and GSS clones. The respective B. napus clone collections were screened by BLASTN search against the ATiDB (The Arabidopsis thaliana Integrated Database) and NCBI (www.ncbi.nlm.nih.gov) databases. The selected EST sequences were used to clone $A B I I$-related genes with gene-specific primer sets and $B$. napus genomic DNA as the template. Genomic DNA was extracted using a DNeasy Plant Mini kit (Qiagen). For full-length cDNA amplification, the Rapid Amplification of cDNA Ends (RACE) method was used using a SMARTer ${ }^{\mathrm{TM}}$ RACE cDNA Amplification Kit (Clontech) according to the manufacturer's instructions. All primer sequences are listed in Supplemental Table S1. The resulting PCR amplicons for genomic and full length cDNA sequences corresponding to individual paralogues were subcloned into StrataClone ${ }^{\mathrm{TM}}$ PCR Cloning Vector pSC-A (Agilent Technologies) and confirmed by sequencing. The NCBI Conserved Domain Database (http://www.ncbi.nlm.nih.gov/Structure/cdd/ wrpsb.cgi) was used to identify the PP2C catalytic domain and characteristic motifs within deduced BnaABI1 protein sequences.

\section{Phylogenetic analysis of $A B I 1$-related genes and other group A PP2Cs in A. thaliana and B. napus}

The deduced group A PP2C amino acid sequences of $B$. napus (Brassica Database; http://brassicadb.org/brad/) were used to estimate their phylogenetic relationships. The clone numbers corresponding to individual PP2C are listed in Supplemental Table S2. First, full-length protein sequences were aligned using the ClustalX program with default parameters. Selected amino acid sequences of the conserved PP2C catalytic domain were further imported into MEGA5.1 software (http://www.megasoftware. net/; Tamura et al. 2011). The phylogenetic tree was constructed using the neighbor-joining method with a Poisson model of amino acid substitution and 1000 bootstrap replications.
Plasmid construction for $B$. napus transgenic plants overexpressing AtABII

For B. napus transformation, the coding region of the AtABII gene (At4g26080) was PCR-amplified using gene-specific primer pairs (Table S1). The amplified AtABII coding sequence was cloned into the pKGIB vector between the BamHI and XhoI sites under the control of a 35S-CaMV promoter (Cegielska-Taras et al. 2008). The construct was introduced into B. napus via Agrobacteriummediated transformation using the method described by Cegielska-Taras et al. (2008). Putative plant transformants were screened on $1 / 2 \mathrm{MS}$ media including $10 \mathrm{mg} / \mathrm{L}$ phosphinotricin. T2 homozygous plants were used for further analysis.

\section{Measurements of physiological parameters}

The chlorophyll and carotenoid contents were determined as described in Ludwików et al. (2009). Ten plants per line were evaluated and all tests were repeated three times.

\section{RNA isolation and qPCR analysis}

RNA isolation and quantitative real-time PCR (qPCR) was performed as described by Ludwików et al. (2013). Total RNA was extracted from three-week old leaves of the WT and transgenic plants using TRIZOL reagent (Invitrogen) following the manufacturer's instructions. For quantitative RT-PCR analysis, $1.5 \mu \mathrm{g}$ DNase-treated RNA was used for the first-strand cDNA synthesis using a First Strand cDNA Synthesis Kit (Fermentas, Gdańsk, Poland) according to manufacturer's protocol. $2 \mu \mathrm{L}$ of 10 -fold diluted cDNA was used as the template in a $15 \mu \mathrm{L}$ qRT-PCR reaction using Maxima SYBR Green/ROX/qPCR Master Mix (Fermentas). An 18S rDNA gene was used as the internal control to normalize all data. Each sample was set up with three biological replicates and run twice on separate plates. PCR reactions were conducted in the ABI PRISM 7900 HT sequence detection system (Applied Biosystems). The qRT-PCR amplifications were carried out using the following thermal profile: $50{ }^{\circ} \mathrm{C}$ for $2 \mathrm{~min}, 95^{\circ} \mathrm{C}$ for $10 \mathrm{~min}$, followed by 40 cycles of $95^{\circ} \mathrm{C}$ for $15 \mathrm{~s}$ and $60^{\circ} \mathrm{C}$ for $1 \mathrm{~min}$. Data analysis was performed using SDS 2.2.1 software (Applied Biosystems). Serial dilutions of genomic DNA (from 100 to 10,000 copies) were used to set up the calibration curve. The results presented here are the mean of the relative gene expression ratios $[\log 2$ fold change scale; $\mathrm{FC}=\log 2$ (ratio/ratio) $]$ of two independent experiments $(n=6)$. After the PCR, a melting curve was generated to test the amplicon specificity. Primer sequences are listed in Supplemental Table S1. 


\section{Promoter activity assay}

The regions almost $2.2 \mathrm{~kb}$ upstream of the translation start codon for both the BnaA01.ABI1.a and BnaC07. $A B I 1 . b$ genes were isolated using the GenomeWalker kit (Clontech) following the manufacturer's instructions. Gene-specific primers for the nested PCR are listed in Supplemental Table S1. For the promoter activity assay, the promoter sequences of the selected BnaABIl paralogues were subcloned into the pENTR/SD/TOPO cloning vector and confirmed by sequencing. To produce plant transformants, $p E N T R$ vector-promoter constructs were recombined with the pMDC162 (::GUS) vector using Gateway ${ }^{\circledR}$ LR Clonase $^{\circledR}$ II Enzyme Mix (Invitrogen). The promoter-reporter fusion construct was transformed into Agrobacterium tumefaciens strain LBA4404 and used for A. thaliana Columbia line plant transformations using the floral dip method (Clough and Bent 1998). Putative plant transformants were screened on $1 / 2 \mathrm{MS}$ media including $10 \mathrm{mg} / \mathrm{L}$ hygromycin. For histochemical analysis of GUS expression, tissue samples were immersed in GUS staining solution $(100 \mathrm{mM}$ sodium phosphate, $\mathrm{pH} 7.0,10 \mathrm{mM}$ EDTA, $0.5 \mathrm{mM}$ $\mathrm{K}_{4} \mathrm{Fe}[\mathrm{CN}]_{6}, 0.5 \mathrm{mM} \mathrm{K}_{3} \mathrm{Fe}[\mathrm{CN}]_{6}, 0.1 \%$ Triton X-100 and $1 \mathrm{mM} \mathrm{X}$-gluc) overnight at $37{ }^{\circ} \mathrm{C}$. After staining, plant samples were rinsed and photographed.

\section{Identification of BnaA01.ABI1. $a$ and BnaC07.ABI1.b cis-regulatory elements}

Potential regulatory elements were identified within the 2,180 bp upstream of the BnaA01.ABI1.a and BnaC07. $A B I 1 . b$ start codons using the MathInspector platform (Genomatix; http://www.genomatix.de/) with the Plant IUPAC Library Version 7.0 (based on PLACE Release 30.0 ) restricted to A. thaliana and IUPAC search parameters and max. $0 \%$ mismatches. Additionally, stressresponsive regulatory motifs were identified by searching the publicly available cis-acting element databases such as Plant Matrix Family Library (Genomatix) and PlantCare (http://bioinformatics.psb.ugent.be/webtools/ plantcare/html/). "Conserved Non-Coding Sequences", named "CNSs", including conserved transcription factor binding sites within the BnaA01.ABI1.a and BnaC07. $A B I 1 . b$ promoters, were defined by sequence similarity (>70 \% identity over at least $90 \mathrm{bp}$ ). Furthermore, these highly conserved regions were analyzed for the presence of conserved stress-responsive cis-regulatory elements using Plant IUPAC Library Version 7.0 on the MathInspector platform. Regions conserved between both promoters were visualized using VISualization Tool for Alignments (Vista) tools mVista and rVista (http://rvista. dcode.org/).

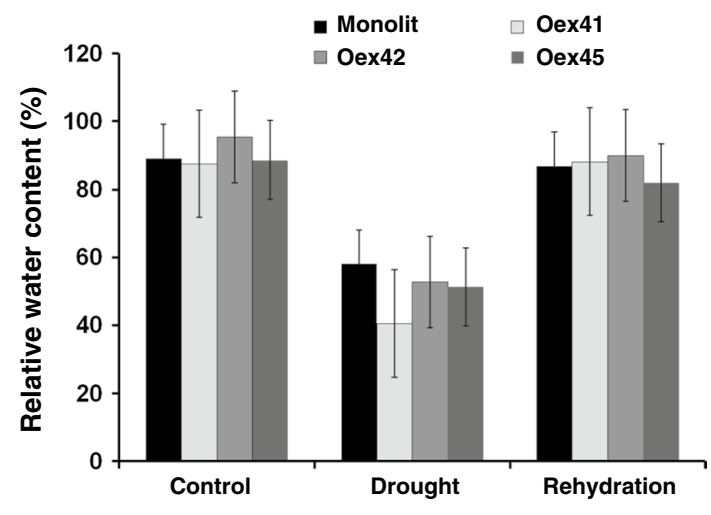

Fig. 1 Relative water content (RWC) in B. napus wild-type and transgenic plants during drought and rehydration. The data represent means from five replicates with three biological repeats. Error bars represent $\mathrm{SE}$

\section{Results}

\section{Response of AtABI1-overexpressing transgenic $B$. napus lines to drought stress: effect on RWC and photosynthetic pigment levels}

To investigate the potential conservation of $\mathrm{ABI} 1$ protein phosphatase 2C function in B. napus, AtABII-overexpressing $B$. napus lines under the control of a 35S-CaMV promoter were generated. Six independent transgenic plants were produced in which the presence of the transgene was confirmed by PCR assay with NOSt/BAR-specific primers (not shown). Three representative lines containing the 35S:AtABI1 construct were used in subsequent experiments (Fig. S1). The transgenic plants were not morphologically different to wild type plants under normal growth conditions (not shown). Because of the involvement of the $A B I 1$ gene in the abiotic stress response (Leung et al. 1997; Chinnusamy et al. 2004), the effect of AtABII overexpression in B. napus plants during drought and rehydration was analyzed. Water status (determined as RWC. i.e. relative water content in leaves) and changes in photosynthetic pigment content were examined in both wild-type plants and transgenic lines grown under well-watered and water-deficient conditions. Measurements of water loss showed no significant differences in RWC between the B. napus control and transgenic plants under normal growth conditions (Fig. 1). However, water content in the leaves of transgenic plants decreased more rapidly during soil drying compared to wild-type plants. In particular, drastic water loss in the leaves of transgenic lines Oex41 (Overexpression line 41) and Oex42 were observed, where RWC decreased by at least $40 \%$. This demonstrates that overexpression of the AtABII orthologue in B. napus plants reduces their water retention capacity, confirming ABI1 role as ABA negative regulator. 
Fig. 2 Total chlorophyll content, chlorophyll $a$, chlorophyll $b$ and chlorophyll $a: b$ ratio in wild type and $A B I 1$-overexpressing transgenic plants under drought conditions. K: control (well-watered) conditions, D: drought conditions. The data represent means from five replicates with three biological repeats. * indicates $P<0.05$ between control and experiment (drought); ** indicates $P<0.05$ between the wild type (Monolit) and the $A B I 1$-overexpressing lines by $t$ test. Error bars indicate SE

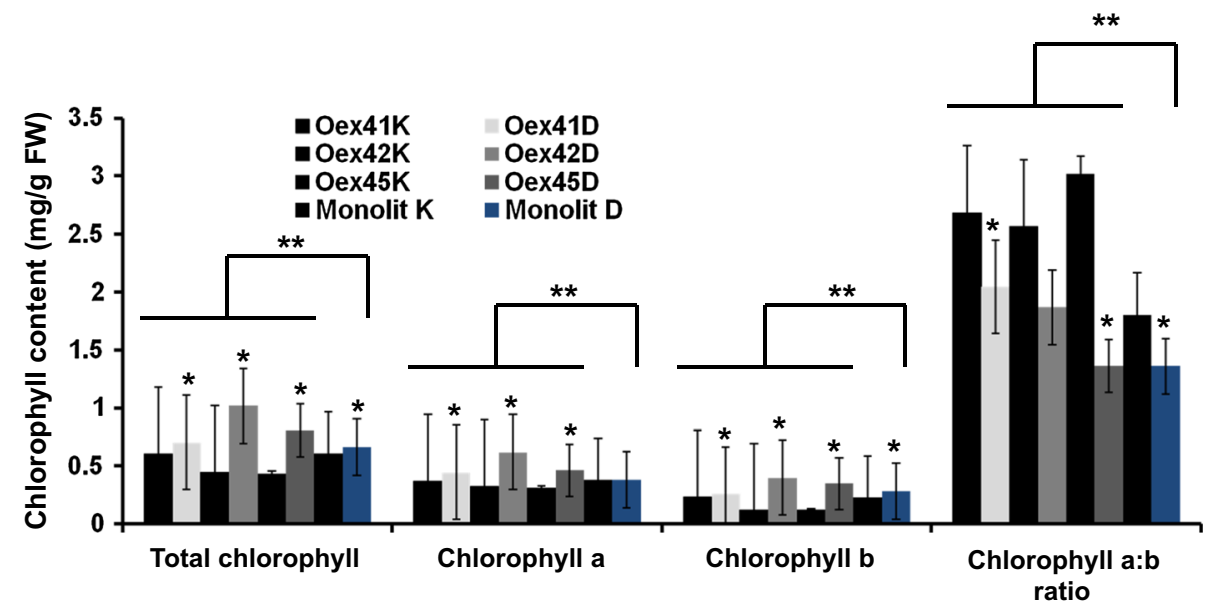

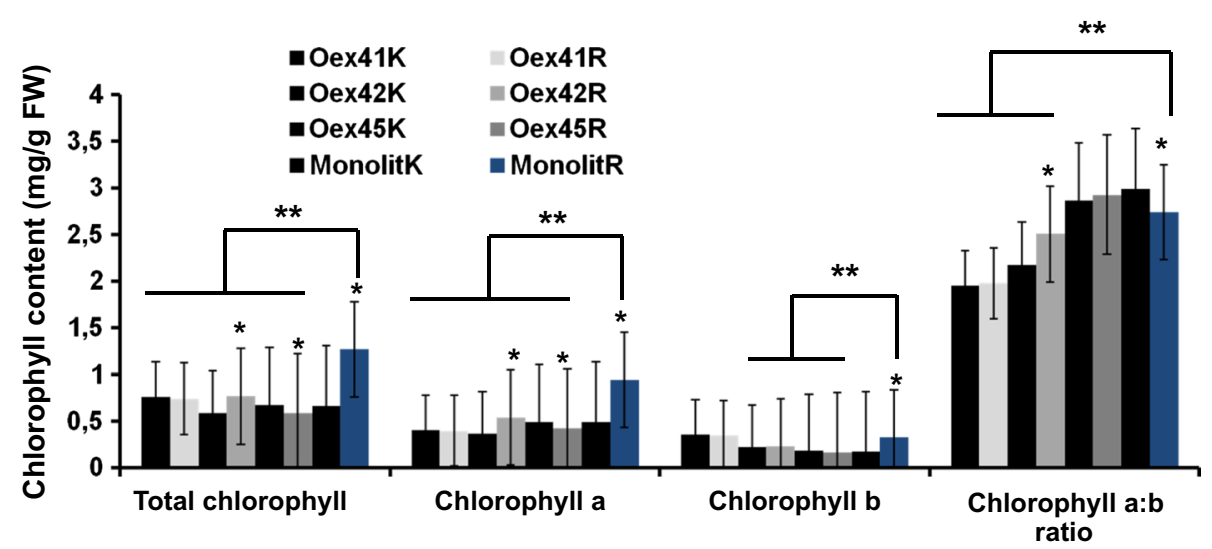

Fig. 3 Total chlorophyll content, chlorophyll $a$, chlorophyll $b$ and chlorophyll $a: b$ ratio in the wild type (Monolit) and ABIl-overexpressing plants after rehydration. K: control (well-watered) conditions, R: drought-treated samples after rehydration. The data rep- resent means from five replicates with three biological repeats. * indicates $P<0.05$, between control and experiment (rehydration); ** indicates $P<0.05$ between wild type and $A B I 1$-overexpressing lines by $t$ test. Error bars indicate SE
These data were complemented by assessment of the content of photosynthetic pigments such as chlorophyll and carotenoids in these plants under well-watered and drought-stress conditions. Initially, chlorophyll $a$, chlorophyll $b$, total chlorophyll content and chlorophyll $a / b$ ratio were analyzed. Changes in photosynthetic pigment content were observed in both wild-type plants and transgenic lines under drought-stress conditions (Fig. 2). As expected, chlorophyll $a$, chlorophyll $b$ and total chlorophyll contents significantly increased in all $B$. napus transgenic lines overexpressing AtABI1. Chlorophyll $a$ levels were higher in all transgenic plants than in wild-type plants, while remaining unchanged in wild-type plants under both drought-stress and control conditions. Chlorophyll $b$ content remained almost invariable in transgenic line Oex41 and in wildtype plants, while its content in lines Oex42 and Oex45 increased and was higher than in wild-type plants. In general, chlorophyll $a$ levels were higher than chlorophyll $b$ levels in both types of plant. However, the chlorophyll $a / b$ ratio decreased in both wild-type plants and the transgenic lines under drought-stress conditions. The largest decrease in chlorophyll $a / b$ ratio was observed in transgenic line Oex45.

Less variation in the photosynthetic pigment content was observed during rehydration conditions (Fig. 3), but there were, nevertheless, slight differences between particular $B$. napus transgenic lines overexpressing AtABI1. Line Oex41 showed no changes in chlorophyll $a$, chlorophyll $b$ and total chlorophyll contents rehydration compared to controls. Chlorophyll $b$ content was invariable in all transgenic lines, but increased in wild type plants under rehydration compared to control, well-watered conditions. On the other hand, total chlorophyll and chlorophyll $a$ contents increased and slightly decreased in lines Oex42 and Oex45, respectively. The chlorophyll $a / b$ ratio increased slightly in all 
Fig. 4 Total carotenoid content of the wild-type (Monolit) and 35S:ABI1 lines under drought and rehydration conditions. $\mathrm{K}$ : control (well-watered) conditions, D: drought conditions. The data represent means from five replicates with three biological repeats. $* *$ indicates $P<0.05$, between control and experiment (drought); * indicates $P<0.05$ between wild type and $A B I 1$ overexpressing lines by $t$ test. Error bars indicate SE

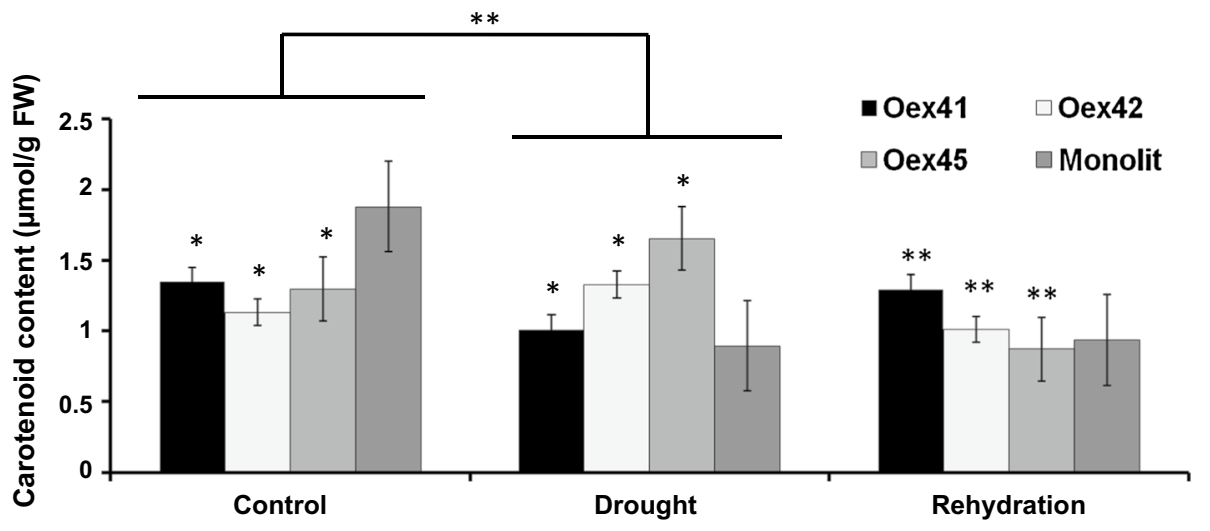

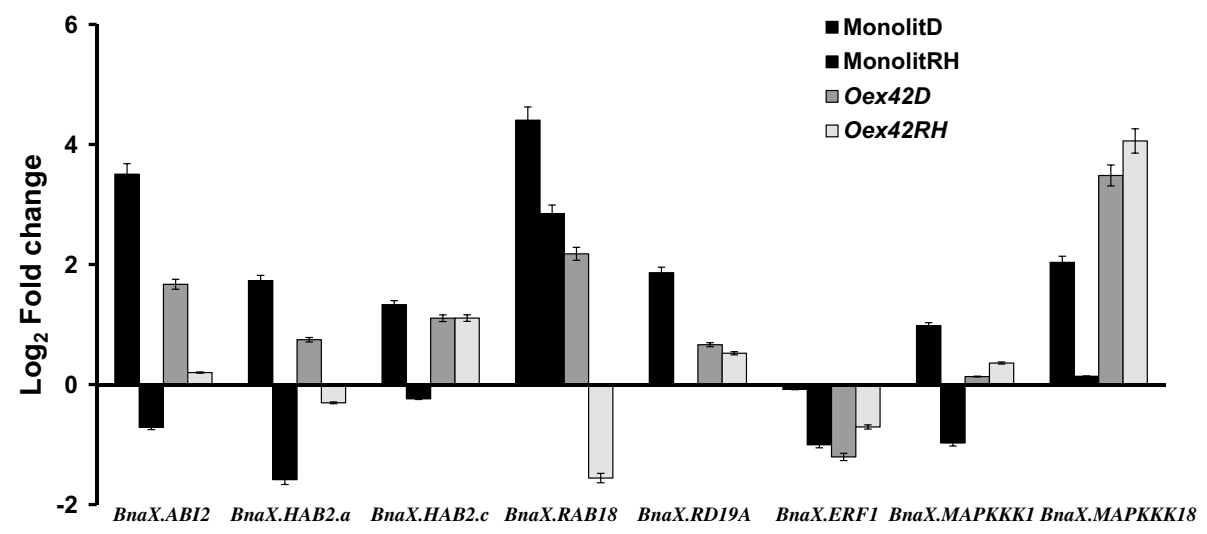

Fig. 5 The relative expression of selected ABA-responsive and drought-responsive genes in wild-type plants and AtABI1-overexpressing B. napus transgenic lines during drought and after rehydration. The qPCR results show relative expression of the indicated genes. Gene transcript levels were determined using three replicates

transgenic lines and decreased in the wild-type plants on rehydration.

Analysis of carotenoid content during drought stress showed a decrease in both wild-type plants and line Oex41, but an increase in lines Oex42 and Oex45 compared to their respective controls (Fig. 4). On rehydration, carotenoid content returned to the control level in transgenic lines Oex41 and Oex42, but decreased in line Oex45 and wild type plants.

\section{Expression of selected ABA-responsive and drought-responsive genes in WT and transgenic $A B I 1-o v e r e x p r e s s i n g ~ l i n e s ~ o f ~ B$. napus}

A set of ABA- and/or stress-inducible genes, as well as other components of the ABA signaling pathway selected from publicly available microarray data for A. thaliana (https://www.genevestigator.com/gv/plant. jsp), were included in our analysis. Among them were genes reported to be inducible by exogenous ABA and/ and were normalized against $18 \mathrm{~S}$ rDNA. Each quantification was repeated at least twice with similar results. The results are displayed as mean $\log 2$ fold change \pm SE $(n=9)$ of three independent experiments

or drought such as RAB18, RD19A and MAPKKK18, other members of the PP2C gene family such as $A B I 2$, two copies of $H A B 2$ and genes induced by other factors such as MAPKKKI (cold and heat responsive) and ERF1 (ethylene responsive). As shown in Fig. 5 for wild type plants and representative transgenic line Oex42, the transcript levels of all genes with the exception of BnaX. ERF1, BnaX.HAB2.c and BnaX.MAPKKK1 increased significantly in wild-type plants and line Oex42 overexpressing $A t A B I 1$ in response to drought stress. However, these genes with the exception of BnaX.MAPKKK18 were expressed at a low level in line Oex42. The expression level of most of these genes returned to control values in both wild-type and transgenic plants under rehydration conditions. Interestingly, the two copies of BnaX.HAB2 studied showed different expression profiles, with only one copy being significantly induced under drought stress. This indicates functional divergence of the duplicated $B n a H A B 2$ genes as an example of gene subfunctionalisation in the ABA signaling network. 
Taken together, these results confirmed that BnaABII genes are negative regulators of the drought response in B. napus. Overexpression of AtABII in the B. napus transgenic lines results in decreased tolerance to drought stress.

\section{Structural characteristics and phylogenetic analysis of the $A B I 1$ paralogues in $B$. napus}

Using the Arabidopsis AtABII cDNA to query the ATiDB and NCBI databases, six $A B I 1$-related genes were identified in the amphidiploid genome of $B$. napus. They are paralogous $A B I 1$ copies, which result from the duplication after last speciation event. These six BnaABII family members were cloned using paralogue-specific primers and then verified by sequencing (Table S1). They were named according to the nomenclature system proposed for the Brassica genus (Østergaard and King 2008). All $B n a A B I 1$-related genes were structurally characterized and their chromosomal localization in the B. napus genome was determined (Babula-Skowronska et al., unpublished). To confirm homology between the $A B I 1$ family members in $B$. napus and their diploid B. rapa and B. oleracea progenitors, a phylogenetic tree was built with high bootstrap support (Fig. S2). This analysis was performed using amino acid sequences of the conserved PP2C catalytic domain for six, three and three ABI1 sequences from $B$. napus, $B$. rapa and $B$. oleracea, respectively. Additionally, the previously detected ABI1s from B. oleracea (KF577723, corresponding to BolC08.ABI1.c; Yuan et al. 2013) and B. napus (JX122895, corresponding to BnaC01.ABI1.a; Zhang et al. 2014) were included. As expected, there was a high degree of similarity between the $A B I 1$ orthologues derived from the $B$. napus, B. rapa and B. oleracea genomes, while the paralogues within the same genomes of the individual species showed greater sequence divergence. A phylogenetic analysis of group A PP2Cs of A. thaliana and B. napus also showed that they divided into two subgroups with high bootstrap support. Moreover, the close evolutionary relationship between the BnaA01.ABII.a and AtABII genes was confirmed based on protein sequence alignment of the PP2C catalytic domain (Fig. S3). These results suggest close evolutionary relationships between the respective $A$. thaliana and B. napus genes.

To investigate the possibility of a functional plasticity of the duplicated BnaABII gene copies in response to environmental stress conditions, two evolutionarily distant BnaABI1 genes, BnaA01.ABII.a and BnaC07.ABII.b, were chosen for subsequent experiments. The first of these represents the closest homologue to A. thaliana AtABII, whereas the second is a more distant homologue of AtABII, dating from the whole-genome duplication that took place after the Arabidopsis and Brassica lineages diverged (Fig. S2). By screening B. rapa (donor of A genome) and B. oleracea (donor of $\mathrm{C}$ genome) genomic DNA using BnaABIl paralogue-specific primers, BnaA01.ABI1.a and BnaC07.ABI1.b were assigned to chromosome $\mathrm{A} 01$ in the $\mathrm{A}$ genome and chromosome $\mathrm{C} 07$ in the $\mathrm{C}$ genome, respectively (Fig. S4). Detailed analysis of both BnaABII genomic and cDNA sequences revealed a highly conserved gene structure with four exons and three introns (Fig. S5). The lengths of the BnaA01.ABII.a and BnaC07.ABII.b genomic sequences are 1588 and $1519 \mathrm{bp}$, coding sequences are 1281 and $1233 \mathrm{bp}$ and deduced protein sequences are 426 and 410 aa, respectively. The sequence similarity of both BnaABII paralogues ranges from 78.6 up to $84.4 \%$ at the genomic and coding sequence levels, respectively. Major sequence differences between both BnaABII genes were discovered in the 5'-UTR, first exon and all introns (Fig. S6). As shown in Fig. S6, the most frequent sequence changes between the two BnaABII genes are deletion/insertion (indels) of longer fragments. For example, one of the longer indels, which includes a microsatellite motif (CAT), is located in the first exon; additional differences occur in sequences flanking this repeat motif. However, the lengths of exon II, exon III and exon IV, 291, 106 and 359 bp, respectively, were maintained in both BnaABII paralogues. To confirm that the selected BnaABII genes belong to the PP2C-type phosphatase gene family, the presence of the PP2C catalytic domain in the deduced protein sequences was determined. Both BnaABI1 proteins contained the PP2C catalytic domain at the $\mathrm{C}$-terminus with the same arrangement of 11 motifs; the N-terminal noncatalytic region was variable in both proteins (Figs. S3 and S7).

\section{Expression of $A B I 1$-related endogenes in transgenic $A B I 1$-overexpressing and WT lines of $B$. napus exposed to drought stress}

The plasticity of ABA signaling in response to stress in polyploids or paleopolyploids can be addressed by investigation of the paralogous $A B I I$ endogenes in B. napus. We analysed their specificity and functional divergence by examining their involvement in the response to drought. We also asked whether AtABII overexpression in the $B$. napus genetic background resulted in co-regulation of transcriptional activity of the endogenous $B n A B I I$ genes. The expression profiles of selected BnaABII endogenes were determined using a qPCR assay in wild-type and transgenic plants under control, drought and rehydration conditions (Fig. 6).

First, to identify any functional redundancy or divergence within the BnaABII paralogues, two evolutionarily distant examples described above (BnaA01.ABI1.a and BnaC07.ABI1.b), were used. Both genes showed different expression patterns during drought stress in WT and in the transgenic plants generated (Fig. 6). Under 
(A)

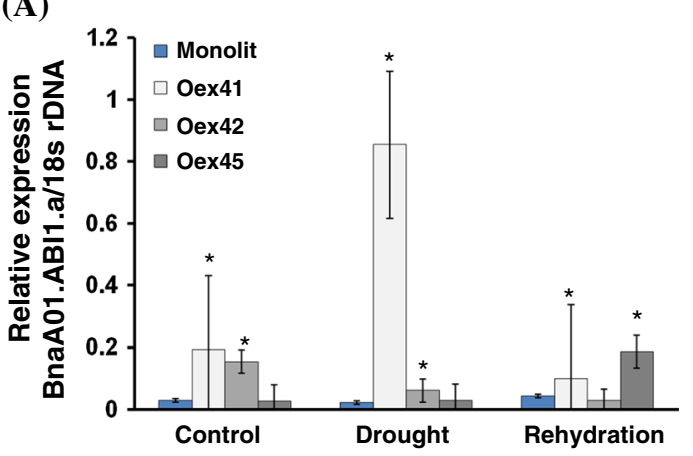

(B)

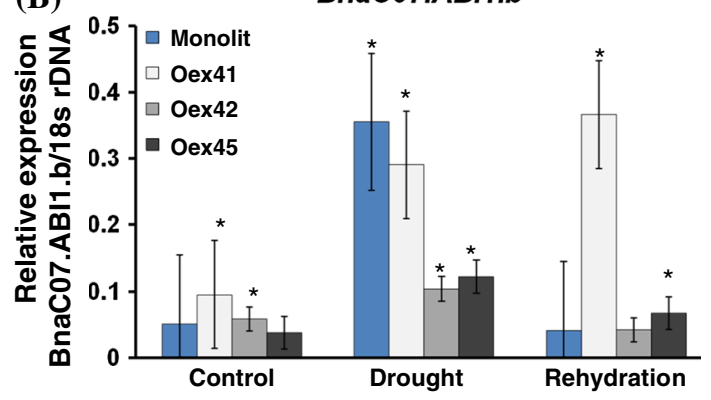

Fig. 6 Relative expression of BnaA01.ABI1.a and BnaC07.ABI1.b genes in wild type and $A B I 1$-overexpressing lines. Results were normalized against $18 \mathrm{~S}$ rDNA expression. Data are representative of three independent experiments. Values represent mean $\pm \mathrm{SE}$

well-watered conditions, transcript levels of both BnaA01. $A B I 1 . a$ and BnaC07.ABII.b in WT plants and transgenic lines were low. However, significant changes in the induction of these genes were observed when the plants were subjected to drought stress. In general, BnaA01.ABI1.a expression did not change in either wild-type or transgenic plants, and remained suppressed; an exception was Oex41, which showed a significant increase in AtABII (and possibly BnaA01ABI1.a) expression compared to the wild-type plants. In contrast, BnaC07.AB11.b was upregulated in both wild-type plants and all transgenic lines exposed to drought. Following rehydration, the expression of BnaC07. $A B I 1 . b$ returned to control levels in wild-type plants and transgenic lines (Fig. 6).

\section{Analysis of BnaA01.ABI1.a and BnaC07.ABI1.b promoter activity}

To further investigate the expression patterns of the BnaA01.ABII.a and BnaC07.ABII.b genes, their $2.2 \mathrm{~kb}$ promoter fragments were fused to the GUS reporter gene and the resulting ProBnaA01.ABI1.a:GUS and ProBnaC07.ABI1.b:GUS constructs were used to generate transgenic $A$. thaliana plants. Five independent transgenic lines for each construct were tested for GUS activity. All construct-specific lines showed the same expression pattern under any conditions tested. Histochemical staining of plants harboring the ProBnaA01.ABI1.a:GUS and ProBnaC07.ABI1.b:GUS constructs showed that there were no major differences in GUS activity in different organs (Fig. 7). Both promoters induced GUS activity in leaves, flowers, siliques and pollen (Fig. 7c-f) and in response to exogenous ABA treatment and wounding (Fig. 8). Interestingly, only BnaC07.ABII.b was induced in the leaves and stems during drought (Fig. 8). This observation confirmed the earlier findings indicating different expression patterns of two BnaABIl paralogues under drought stress conditions (Fig. 6). To better understand transcriptional regulation of the BnaA01.ABI1.a and BnaCO7. $A B I 1 . b$ genes, a de novo search for ABA- and water deficit-responsive cis-regulatory elements within the $2.2 \mathrm{~kb}$ regions upstream of their translation start codons was performed. Based on data from different databases (PLACE, Plant Matrix Family Library at Genomatix platform and PlantCare) it was shown that the BnaA01.ABIl.a and BnaC07.ABII.b promoters contained 20 and 14 ABAand osmotic stress-responsive cis-elements within the $2.2 \mathrm{~kb}$ upstream of the translation start site, respectively (Table S3). They include known cis-acting plant elements such as ABREs, MYB recognition sites, NYC recognition sites and SALTs, which are arranged in several copies throughout the upstream regions. The sequence similarity between the BnaA01.ABII.a and BnaC07.ABII.b promoter regions is $56.1 \%$ which as expected is much lower than between their protein-coding sequences (Fig. S8). Comparison of both promoter sequences allowed us to identify six conserved regions with almost $70 \%$ sequence similarity over at least 90 bp (Figs. 9 and S8). These regions covered over $52 \%$ of each promoter and included some common regulatory elements located at similar relative positions. Detailed comparative studies of regulatory motifs involved in abscisic acid responsiveness such as ABRE, NAC, MYB and SALT revealed variations in their numbers and genome localization in the two promoter regions (Table S3; Fig. S8). Only four common protein-binding elements were identified in the conserved regions (Fig. 9). These data imply that sequence variation in the BnaA01. $A B I 1 . a$ and BnaC07.ABII.b promoter regions, outside the conserved regions, may be responsible for changes in their induction under drought stress (Figs. 6 and 8). Especially that some of drought-responsive elements such as MYB were identified in 4 copies within $600-800$ bp promoter region of drought-inducible BnaC07.ABI1.b paralogue; meaningfully, drought-insensitive BnaC01.ABI1.a paralogue misses that regulatory boxes in the corresponding promoter region. 

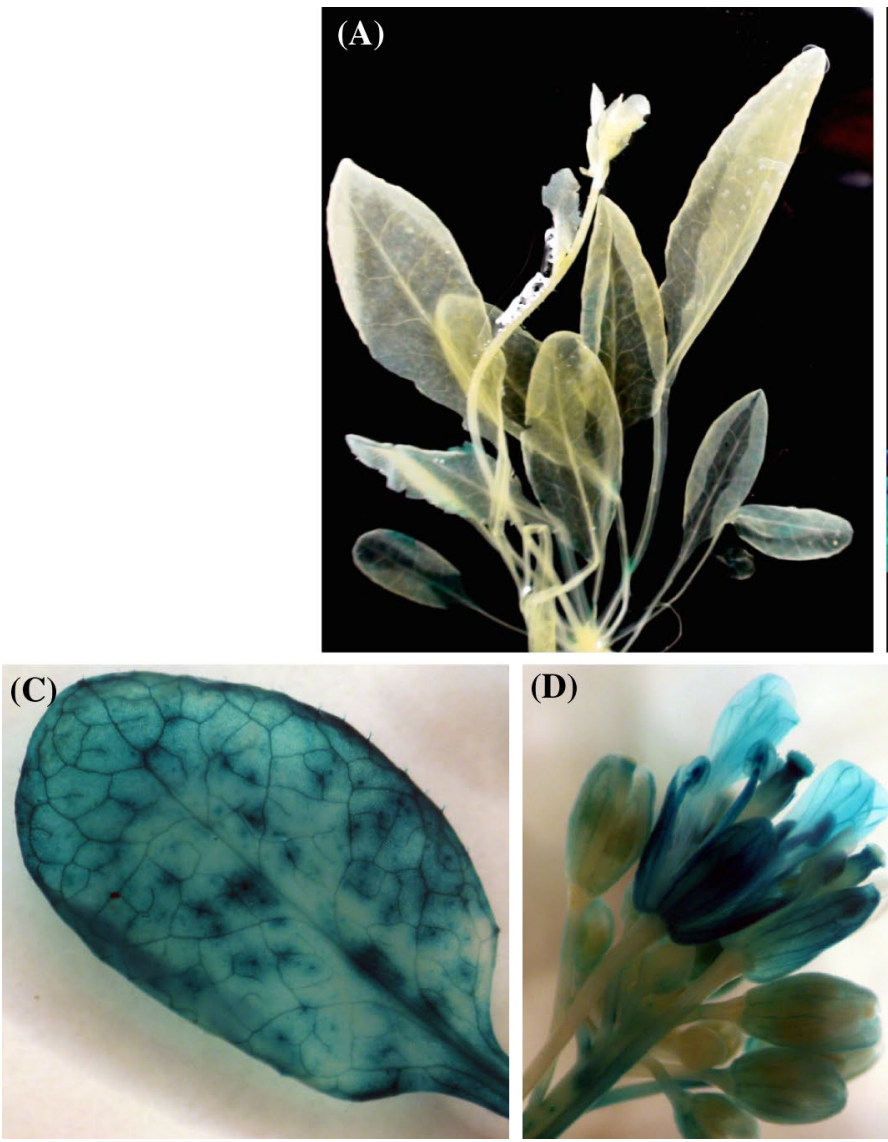

(D)

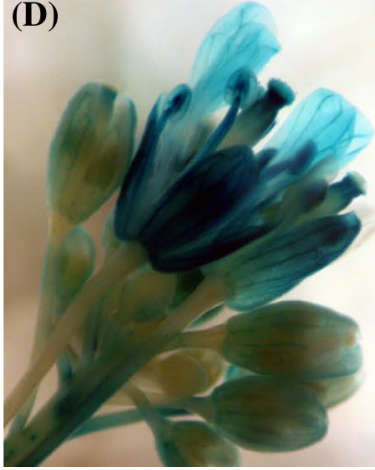

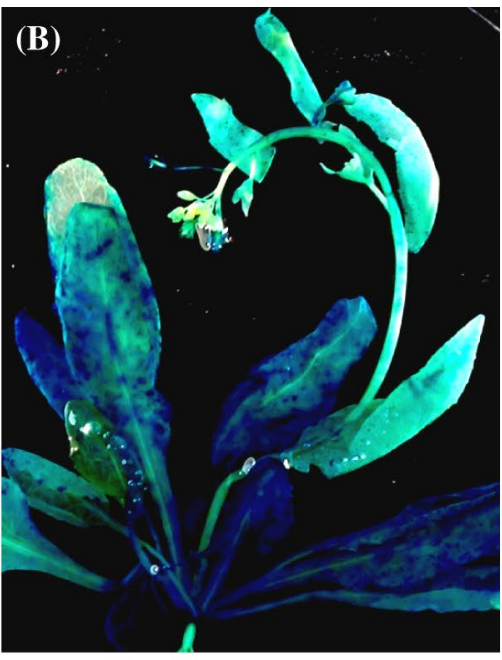

(E)

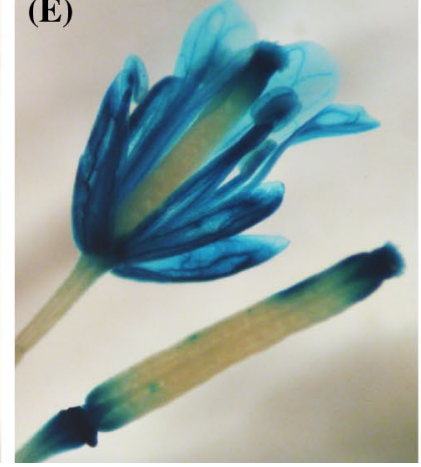

(F)

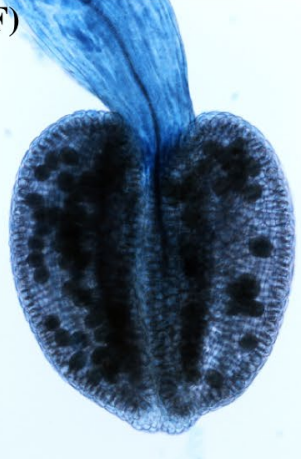

Fig. 7 Localization of ProBnaA01.ABI1.a:GUS and ProBnaC07. ABI1.b:GUS activity (blue staining) in transgenic $A$. thaliana plants. A-mock, B-whole plant, C-leaves, D-flowers, E-siliques, F-pollen.
Experiments were repeated $(\mathrm{n}=3)$ with similar results and representative data are shown
Fig. 8 Histochemical visualization of GUS activity (blue staining) directed by BnaA01.ABI1.a and BnaC07.ABI1.b upstream regions in A. thaliana transgenic plants. Experiments were repeated $(n=3)$ with similar results and representative data are shown
ProBnaA01.ABI1.a:GUS

ProBnaC07.ABI1.b:GUS

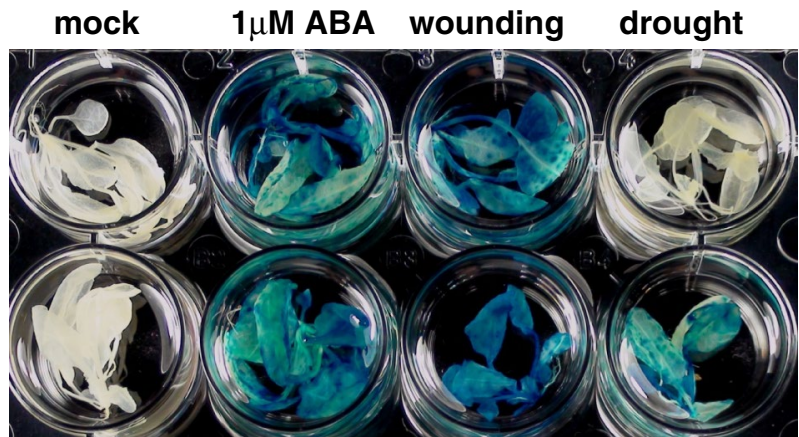

\section{Discussion}

In this report, we show that the role of the $A B I 1$ orthologous gene (unique gene copy from the Arabidopsis genome) is conserved in Brassicaceae. We investigated the effect of $A t A B I I$ overexpression in B. napus in drought stress conditions. Our results on the role of ABA signaling in drought stress, in particular the involvement of ABI1 protein phosphatase, highlight the complexity of the $B$. napus stress response mechanism. The transgenic B. napus lines overexpressing AtABII were characterized in detail with respect to their drought stress response. When overexpressed in B. napus, AtABII negatively influences several important cellular processes such as plant water content (for example, line Oex41, which has a high ABII transcript level, suffered water loss of $40 \%$ ), chlorophyll accumulation and the expression profile of several ABA- and/ or dehydration stress-inducible genes (Figs. 1, 2 and 5). 


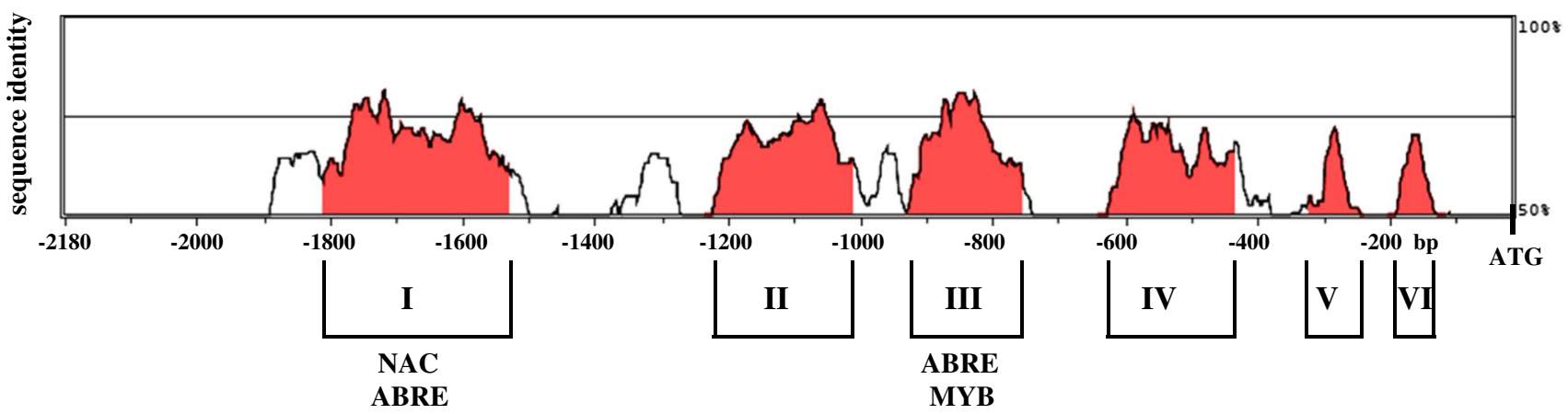

Fig. 9 Conservation of the BnaA01.ABI1. $a$ and BnaC07.ABI1.b promoter regions in $B$. napus. Six conserved regions named "Conserved Non-Coding Sequences" were defined by sequence similarity at

Intriguingly, most genes with the exception of BnaA01. ABII.a and BnaX.MAPKKK18 were transcriptionally induced in control plants in a similar way to counterparts in A. thaliana. The expression profiles of selected PP2Cs, such as BnaA01.ABI1.a, BnaC07.ABI1.b, BnaX.ABI2, BnaX.HAB2.a and BnaX.HAB2.c, were also similar to those found in $B$. oleracea exposed to drought stress (Ludwików et al. 2013). Thus, our results highlight the regulatory functions of the ABI1 PP2C, which is highly conserved within the Brassicaceae family, and suggest that it plays a role as a regulatory hub protein (for review Ma et al. 2009) for dehydration stress responses in plants. Our results showing that the expression levels of several ABA-regulated genes were reduced in each transgenic line exposed to drought stress are consistent with such a role for ABI1 as a regulator of the drought stress response. Our previous results, which showed that $\mathrm{ABI} 1$ negatively regulates expression of $R A B 18, M A P K K K 18$ and other drought-induced genes (Ludwików et al. 2009), supports this hypothesis.

Understanding the biological role of duplicated genes, and particularly whether they have redundant or divergent functions in stress responses, is currently a high priority, although it is a challenging task in crop plant studies. We demonstrate differential involvement of the B. napus $A B I 1$ paralogues in the water-stress response. There are six $A B I 1$-related genes in the $B$. napus genome, consistent with previous genetic studies indicating the presence of several ABII gene copies in the Brassica genomes (Sadowski and Quiros 1998; Quiros et al. 2001; Ludwików et al. 2013). By analysis of the genome sequences of $B$. rapa and B. olerace $a$ and of different sets of B. napus EST clones, the six copies disclosed represent the complete set of BnaABII paralogues in B. napus. Prior to the current work, single $A B I 1$-like gene had been described in the $B$. napus genome, corresponding to that identified and characterized herewith as BnaC01.ABI1.a (Zhang et al. 2014; Fig. S2). As expected, a phylogenetic analysis of group A the level of $>70 \%$ over a $90 \mathrm{bp}$ interval. The sequences conserved between both promoters were visualized using VISualization Tool for Alignments (Vista) tools mVista and rVista (http://rvista.dcode.org/)

PP2Cs in A. thaliana and B. napus confirmed their close evolutionary relationship (Fig. S2). Generally, the number of multiple copies of BnaABII correspond proportionally to the increase in total gene number in $B$. napus polyploid genome. This suggests that a repertoire of protein targets recognized by numerous BnaABI1 $s$ is enlarged. Despite the difference in the number of genes in both species, they divided into two subgroups as in other species (Xue et al. 2008; Komatsu et al. 2009).

The maintenance of several duplicated copies of key regulatory genes over long periods of evolution is consistent with the gene balance hypothesis, which assumes that elements of complex signaling networks are preferentially protected against gene loss (Blanc and Wolfe 2004; Wang et al. 2011). Certainly, if such duplicated gene copies acquire different functions, they are more likely to be stably retained in a given genome. Studies on different organisms reveal that members of gene families may respond differently to environmental conditions as a result of either sub- and/or neofunctionalization (for example, Whittle and Krochko 2009; Liu and Adams 2010). Using a custom-made Arabidopsis cDNA macroarray, we previously revealed differences in the gene expression profiles of PP2C (group A) members under drought conditions and after ABA treatment in B. oleracea and A. thaliana (Ludwików et al. 2013). Interestingly, the BolABIl gene (detected by AtABII-gene-specific primers) was downregulated during drought stress in B. oleracea; this suggests that it is not essential for the drought stress response. On the other hand, subsequent studies indicate that one of the BolABII genes in B. oleracea corresponding to BnaC08. $A B I 1 . c$ in B. napus is induced following exogenous ABA treatment and in the early stages of drought stress, confirming its involvement in the ABA signaling pathway (Yuan et al. 2013).

To determine the degree of conservation of the transcriptional regulation of the BnaABII paralogues, BnaA01. 
ABII.a and BnaC07.ABII.b, which represent the closest and a more distant homologues of the A. thaliana AtABII gene, these genes were characterized in detail (Fig. S2). Both BnaABIl paralogues have a similar gene structure, with four exons and three introns, a C-terminal PP2C catalytic domain architecture with the same arrangement of 11 motifs, and identical localization of the NLS-like motif (Figs. S3, S5 and S7). Multiple alignments of genomic, cDNA and predicted protein sequences showed a high degree of sequence similarity between BnaA01.ABI1. $a$ and BnaC07.ABI1.b of up to almost $85 \%$. However, our analysis reveals sequence variability in the $5^{\prime}$ upstream regions including the promoter, exon I and intron sequences, enabling gene-specific analyses at both the transcriptional and proteomic levels (Fig. S6). These data confirmed previous results indicating reduced sequence conservation of these upstream regions among members of the same gene family, which may reflect changes in the expression pattern of the respective genes (Chen et al. 2011). Our finding that the transcriptional expression patterns of selected BnaABII endogenous paralogues, i.e. BnaA01.ABI1.a and BnaC07. $A B I 1 . b$, significantly differ in response to drought stress, while showing similar transcriptional patterns after treatment with other stimuli (Figs. 6, 8), is entirely novel. Both genes appeared to be responsive to exogenous ABA, but only BnaC07.ABI1.b is induced by drought. Thus, BnaA01. $A B I 1 . a$ and BnaC07.ABII.b endogenes may have developed new subfunctions that improve the fine-scale response to drought stress. It remains possible that further subfunctions have evolved in other $B$. napus $A B I 1$ and $P P 2 C$ group A homologues. Similarly, the different expression patterns under drought were found for two BnaHAB2 gene copies in WT plants and AtABII overexpressed lines of B. napus, revealing gene subfunctionalisation in the ABA signaling network (Fig. 5). This is a promising area of investigation as a comparative analysis of the $B$. rapa transcriptome (Lee et al. 2008) indicates that $B$. rapa homologues corresponding to BnaA01.ABI1.a and BnaC07.ABI1.b also differ in the response to salt stress: the $B$. rapa homologue of BnaA01. $A B I 1 . a$ was found to be responsive to salt stress while, in contrast, a homologue of BnaC07.ABII.b was not. In light of our observations, we assume that BnaABII homologues are potentially drought- as well as ABA-responsive, as these two stimuli positively induces all ABI1 phosphatases studied so far in different species.

Presently, little is known about the transcriptional regulation of the PP2C gene family in plants. A previous study revealed that the A. thaliana $A B I 1$ promoter is active in guard cells and root meristem (Leung et al. 2006). To approximate a mechanism controlling induction of both BnaABII promoters under ABA treatment and drought stress, in silico identification of ABAinducible and stress-responsive elements within these regulatory regions was performed. Previous studies on the mechanisms of transcriptional regulation of ABAinducible genes identified specific cis-acting elements associated with the responses to ABA, osmotic and water stresses (Narusaka et al. 2003; Yamaguchi-Shinozaki and Shinozaki 2005, 2006). Among the best described are the ABRE (ABA-responsive element), MYB, MYC, NAC, DRE, SALT and W-box elements recognized by the $A R E B / A B F, M Y B, M Y C, N A C, D R E B$, Alphin and $W R K Y$ family transcription factors, respectively (Abe et al. 2003; Yamaguchi-Shinozaki and Shinozaki 2005; Wang et al. 2009). Some reports document the interaction between $A B I I$ and transcription factors in Arabidopsis (Himmelbach et al. 2002; Cui et al. 2013). Thus, AtMYB20, which binds to the MYB recognition sequence (TAACTG) and the ACGT core element in the ABII and $A t P P 2 C A$ promoter regions, acts as a negative regulator of $P P 2 C$ genes, thereby enhancing salt tolerance (Cui et al. 2013). Interestingly, sequence comparison of AtABII and both BnaABII promoter regions showed that these cis-acting elements binding AtMYB20 are only present in the BnaA01.ABI1.a gene (data not shown). This suggests that AtABII and one, at least, of the $B$. napus BnaABI1-like genes may be involved in the same $\mathrm{NaCl}$-stress-responsive pathways. An earlier study also demonstrated physical interaction between the homeodomain transcription factor AtHB6 and ABI1, which was positively correlated with PP2C activity (Himmelbach et al. 2002). To identify potentially all cis-acting elements associated with the response to $\mathrm{ABA}$ and/or drought stress within the BnaA01.ABI1.a and BnaC07. $A B I 1 . b$ promoters, plant databases such as Plant Matrix Family Library, PLACE and PlantCare were used, revealing several ABA-inducible and stress-responsive elements (Table S3). Comparative sequence analysis of the BnaA01.ABII.a and BnaC07.ABI1.b promoters showed significant differences in number and localization of ABA- and stress-inducible regulatory elements, despite the close relationship of these genes (Table S3 and Fig. S8). Interestingly, most elements are located from -1180 to $-1680 \mathrm{bp}$ and from -680 to $-1180 \mathrm{bp}$ in the BnaA01.ABI1.a and BnaC07.ABII.b promoters, respectively, i.e. outside the most conserved regions. Only four of the regulatory motifs detected are located in the CNSs of both promoters (Fig. 9). In summary, comparison of the two promoters suggests that the lack of induction of BnaA01.ABI1.a by drought could result from the loss of several cis-elements in the BnaA01.ABII.a promoter during the course of evolution. This indicates that specific expression patterns of these homologous genes, resulting in their subfunctionalization, may be modulated through mutation and possibly also through epigenetic changes within their respective promoter regions. 
Acknowledgments We thank Assist. Prof. Tomasz Pniewski for the pKGIB vector. This work was supported by the Polish Committee for Scientific Research Grants PBZ-MNiSW-2/3/2006/19/IGR/1, PBZ-MNiSW-2/3/2006/19/IGR/3, MNiSW-2/3/2006/19/IGR/4 and N N303 568339.

Conflict of interest The authors declare that they have no conflict of interest.

Open Access This article is distributed under the terms of the Creative Commons Attribution 4.0 International License (http://creativecommons.org/licenses/by/4.0/), which permits unrestricted use, distribution, and reproduction in any medium, provided you give appropriate credit to the original author(s) and the source, provide a link to the Creative Commons license, and indicate if changes were made.

\section{References}

Abe H, Urao T, Ito T, Seki M, Shinozaki K, Yamaguchi-Shinozaki K (2003) Arabidopsis AtMYC2 (bHLH) and AtMYB2 (MYB) function as transcriptional activators in abscisic acid signaling. Plant Cell 15:63-78

Babula D, Kaczmarek M, Barakat A, Delseny M, Quiros CF, Sadowski J (2003) Chromosomal mapping of Brassica oleracea based on ESTs from Arabidopsis thaliana: complexity of the comparative map. Mol Genet Genomics 268:656-665

Babula D, Misztal LH, Jakubowicz M, Kaczmarek M, Nowak W, Sadowski J (2006) Genes involved in biosynthesis and signalisation of ethylene in Brassica oleracea and Arabidopsis thaliana: identification and genome comparative mapping of specific gene homologues. Theor Appl Genet 112:410-420

Blanc G, Wolfe KH (2004) Functional divergence of duplicated genes formed by polyploidy during Arabidopsis evolution. Plant Cell 16:1667-1678

Bork P, Brown NP, Hegyi H, Schultz J (1996) The protein phosphatase 2C (PP2C) superfamily: detection of bacterial homologues. Protein Sci 5:1421-1425

Cegielska-Taras T, Pniewski T, Szała L (2008) Transformation of microspore-derived embryos of winter oilseed rape (Brassica napus L.) by using Agrobacterium tumefaciens. J Appl Genet 49:343-347

Chen X, Truksa M, Snyder CL, El-Mezawy A, Shah S, Weselake RJ (2011) Three homologous genes encoding sn-glycerol3-phosphate acyltransferase 4 exhibit different expression patterns and functional divergence in Brassica napus. Plant Physiol $155: 851-865$

Chinnusamy V, Schumaker K, Zhu J-K (2004) Molecular genetic perspectives on cross-talk and specificity in abiotic stress signalling in plants. J Exp Bot 55:225-236

Clough SJ, Bent AF (1998) Floral dip: a simplified method for Agrobacterium-mediated transformation of Arabidopsis thaliana. Plant J 16:735-743

Cui MH, Yoo KS, Hyoung S, Nguyen HTK, Kim YY, Kim HJ, Ok SH, Yoo SD, Shin JS (2013) An Arabidopsis R2R3-MYB transcription factor, AtMYB20, negatively regulates type 2C serine/ threonine protein phosphatases to enhance salt tolerance. FEBS Lett 587:1773-1778

Fujita Y, Nakashima K, Yoshida T, Katagiri T, Kidokoro S, Kanamori $\mathrm{N}$ et al (2009) Three SnRK2 protein kinases are the main positive regulators of abscisic acid signaling in response to water stress in Arabidopsis. Plant Cell Physiol 50:2123-2132
Geiger D, Scherzer S, Mumm P, Marten I, Ache P, Matschi S, Liese A, Wellmann C, Al-Rasheid KA, Grill E, Romeis T, Hedrich R (2010) Guard cell anion channel SLAC1 is regulated by CDPK protein kinases with distinct $\mathrm{Ca} 2+$ affinities. Proc Natl Acad Sci USA 107:8023-8028

Gonzalez-Garcia MP, Rodriguez D, Nicolas C, Rodriguez PL, Nicolas G, Lorenzo O (2003) Negative regulation of abscisic acid signaling by the Fagus sylvatica FsPP2C1 plays a role in seed dormancy regulation and promotion of seed germination. Plant Physiol 133:135-144

Gosti F, Beaudoin N, Serizet C, Webb AA, Vartanian N, Giraudat J (1999) ABI1 protein phosphatase $2 \mathrm{C}$ is a negative regulator of abscisic acid signaling. Plant Cell 11:1897-1910

Himmelbach A, Hoffmann T, Leube M, Hohener B, Grill E (2002) Homeodomain protein ATHB6 is a target of the protein phosphatase ABI1 and regulates hormone responses in Arabidopsis. EMBO J 21:3029-3038

Jia H-F, Lu D, Sun J-H, Li C-L, Xing Y, Qin L, Shen Y-Y (2013) Type $2 \mathrm{C}$ protein phosphatase $\mathrm{ABI} 1$ is a negative regulator of strawberry fruit ripening. J Exp Bot 64:1677-1687

Komatsu K, Nishikawa Y, Ohtsuka T, Taji T, Quatrano RS, Tanaka S, Sakata Y (2009) Functional analyses of the ABI1-related protein phosphatase type $2 \mathrm{C}$ reveal evolutionarily conserved regulation of abscisic acid signaling between Arabidopsis and the moss Physcomitrella patens. Plant Mol Biol 70:327-340

Lee SC, Lim MH, Kim JA, Lee SI, Kim JS, Jin M, Kwon SJ, Mun JH, Kim YK, Kim HU, Hur Y, Park BS (2008) Transcriptome analysis in Brassica rapa under the abiotic stresses using Brassica 24 K oligo microarray. Mol Cells 26:595-605

Leung J, Bouvier-Durand M, Morris PC, Guerrier D, Chefdor F, Giraudat J (1994) Arabidopsis ABA response gene ABI1: features of a calcium-modulated protein phosphatase. Science 264:1448-1452

Leung J, Merlot S, Giraudat J (1997) The Arabidopsis ABSCISIC ACID-INSENSITIVE2 ( $A B I 2)$ and $A B I 1$ genes encode homologous protein phosphatases $2 \mathrm{C}$ involved in abscisic acid signal transduction. Plant Cell 9:759-771

Leung J, Orfanidi S, Chefdor F, Mészaros T, Bolte S, Mizoguchi T, Shinozaki K, Giraudat J, Bögre L (2006) Antagonistic interaction between MAP kinase and protein phosphatase $2 \mathrm{C}$ in stress recovery. Plant Sci 171:596-606

Liu SL, Adams KL (2010) Dramatic change in function and expression pattern of a gene duplicated by polyploidy created a paternal effect gene in the Brassicaceae. Mol Biol Evol 27:2817-2828

Ludwików A (2015) Targeting proteins for proteasomal degradation-a new function of Arabidopsis ABI1 protein phosphatase 2C. Front Plant Sci 6:310

Ludwików A, Kierzek D, Gallois P, Zeef L, Sadowski J (2009) Gene expression profiling of ozone-treated Arabidopsis abiltd insertional mutant: protein phosphatase $2 \mathrm{C}$ ABI1 modulates biosynthesis ratio of ABA and ethylene. Planta 230:1003-1017

Ludwików A, Babula-Skowrońska D, Szczepaniak M, Belter N, Dominiak E, Sadowski J (2013) Expression profiles and genomic organisation of group A protein phosphatase 2C genes in Brassica oleracea. Annals Appl Biol 163:124-134

Ludwików A, Cieśla A, Kasprowicz-Maluśki A, Mituła F, Tajdel M, Gałgański Ł, Ziółkowski PA, Kubiak P, Małecka A, Piechalak A, Szabat M, Górska A, Dąbrowski M, Ibragimow I, Sadowski J (2014) Arabidopsis protein phosphatase 2C ABI1 interacts with type I ACC synthases and is involved in the regulation of ozoneinduced ethylene biosynthesis. Mol Plant 7:960-976

Lysak MA, Koch MA, Pecinka A, Schubert I (2005) Chromosome triplication found across the tribe Brassiceae. Genome Res $15: 516-525$ 
Ma Y, Szostkiewicz I, Korte A, Moes D, Yang Y, Christmann A, Grill E (2009) Regulators of PP2C phosphatase activity function as abscisic acid sensors. Science 324:1064-1068

Merlot S, Gosti F, Guerrier D, Vavasseur A, Giraudat J (2001) The $\mathrm{ABI} 1$ and $\mathrm{ABI} 2$ protein phosphatases $2 \mathrm{C}$ act in a negative feedback regulatory loop of the abscisic acid signalling pathway. Plant J 25:295-303

Narusaka Y, Nakashima K, Shinwari ZK, Sakuma Y, Furihata T, Abe H, Narusaka M, Shinozaki K, Yamaguchi-Shinozaki K (2003) Interaction between two cis-acting elements, ABRE and DRE, in ABA-dependent expression of Arabidopsis rd29A gene in response to dehydration and high-salinity stresses. Plant $\mathrm{J}$ 34:137-148

Nemhauser JL, Hong F, Chory J (2006) Different plant hormones regulate similar processes through largely nonoverlapping transcriptional responses. Cell 126:467-475

Østergaard L, King GJ (2008) Standardized gene nomenclature for the Brassica genus. Plant Methods 4:10

Parkin IAP, Gulden SM, Sharpe AG, Lukens L, Trick M, Osborn TC, Lydiate DJ (2005) Segmental structure of the Brassica napus genome based on comparative analysis with Arabidopsis thaliana. Genetics 171:765-781

Quiros CF, Grellet F, Sadowski J, Suzuki T, Li G, Wroblewski $\mathrm{T}$ (2001) Arabidopsis and Brassica comparative genomics: sequence, structure and gene content in the ABI1-Rps2-Ck1 chromosomal segment and related regions. Genetics 157:1321-1330

Reymond P, Weber H, Damond M, Farmer EE (2000) Differential gene expression in response to mechanical wounding and insect feeding in Arabidopsis. Plant Cell 12:707-719

Rubio S, Rodrigues A, Saez A, Dizon MB, Galle A, Kim T et al (2009) Triple loss of function of protein phosphatases type $2 \mathrm{C}$ leads to partial constitutive response to endogenous abscisic acid. Plant Physiol 50:1345-1355

Sadowski J, Quiros CF (1998) Organization of an Arabidopsis thaliana gene cluster on chromosome 4 including the RPS2 gene, in the Brassica nigra genome. Theor Appl Genet 96:468-474

Saez A, Apostolova N, Gonzalez-Guzman M, Gonzalez-Garcia MP, Nicolas C, Lorenzo O, Rodriguez PL (2004) Gain-of-function and loss-of-function phenotypes of the protein phosphatase $2 \mathrm{C}$ $\mathrm{HAB} 1$ reveal its role as a negative regulator of abscisic acid signaling. Plant J 37:354-369

Schweighofer A, Heribert Hirt H, Meskiene I (2004) Plant PP2C phosphatases: emerging functions in stress signaling. Trends Plant Sci 9:236-243

Sheen J (1998) Mutational analysis of protein phosphatase 2C involved in abscisic acid signal transduction in higher plants. Proc Natl Acad Sci 95:975-980

Studer RA, Robinson-Rechavi M (2009) How confident can we be that orthologs are similar, but paralogs differ? Trends Gen 25:210-216

Swamy PM, Smith B (1999) Role of abscisic acid in plant stress tolerance. Current Sci 76:1220-1227

Tamura K, Peterson D, Peterson N, Stecher G, Nei M, Kumar S (2011) MEGA5: molecular evolutionary genetics analysis using maximum likelihood, evolutionary distance, and maximum parsimony methods. Mol Biol Evol 28:2731-2739
Tougane K, Komatsu K, Bhyan SB, Sakata Y, Ishizaki K, Yamato KT et al (2010) Evolutionarily conserved regulatory mechanisms of abscisic acid signaling in land plants: characterization of ABSCISIC ACID INSENSITIVE1-like type 2C protein phosphatase in the liverwort Marchantia polymorpha. Plant Physiol 152:1529-1543

Udall JA, Wendel JF (2006) Polyploidy and crop improvement. Crop Sci 46:S-3-S-14

Umezawa T, Sugiyama N, Mizoguchi M, Hayash S, Myouga F, Yamaguchi-Shinozaki K et al (2009) Type 2C protein phosphatases directly regulate abscisic acid-activated protein kinases in Arabidopsis. Proc Natl Acad Sci USA 106:17588-17593

Umezawa T, Nakashima K, Miyakawa T, Kuromori T, Tanokura M, Shinozaki K, Yamaguchi-Shinozaki K (2010) Molecular basis of the core regulatory network in ABA responses: sensing, signaling and transport. Plant Cell Physiol 51:1821-1839

Wang Z, Zhu Y, Wang L, Liu X, Liu Y, Phillips J, Deng X (2009) A WRKY transcription factor participates in dehydration tolerance in Boea hygrometrica by binding to the W-box elements of the galactinol synthase (BhGolS1) promoter. Planta 230:1155-1166

Wang X, Wang H, Wang J, Sun R, Wu J, Liu S, Bai Y, Mun JH, Bancroft I, Cheng F et al (2011) The genome of the mesopolyploid crop species Brassica rapa. Nat Genet 43:1035-1039

Whittle CA, Krochko JE (2009) Transcript profiling provides evidence of functional divergence and expression networks among ribosomal protein gene paralogs in Brassica napus. Plant Cell 21:2203-2219

Xue T, Wang D, Zhang S, Ehlting J, Ni F, Jakab S, Zheng C, Zhong Y (2008) Genome-wide and expression analysis of protein phosphatase $2 \mathrm{C}$ in rice and Arabidopsis. BMC Genomics 9:550

Yamaguchi-Shinozaki K, Shinozaki K (2005) Organization of cis-acting regulatory elements in osmotic- and cold-stress-responsive promoters. Trends Plant Sci 10:88-94

Yamaguchi-Shinozaki K, Shinozaki K (2006) Transcriptional regulatory networks in cellular responses and tolerance to dehydration and cold stresses. Annu Rev Plant Biol 57:781-803

Yoshida T, Nishimura N, Kitahata N, Kuromori T, Ito T, Asami T, Shinozaki K, Hirayama T (2006) ABA-hypersensitive germination 3 encodes a protein phosphatase $2 \mathrm{C}$ (AtPP2CA) that strongly regulates abscisic acid signaling during germination among Arabidopsis protein phosphatase 2Cs. Plant Physiol 140:115-126

Yuan F, Wang M, Hao H, Zhang Y, Zhao H, Guo A, Xu H, Zhou X, Xie CG (2013) Negative regulation of abscisic acid signaling by the Brassica oleracea ABI1 ortholog. Biochem Biophys Res Commun 442:202-208

Zhang H, Liu W-Z, Zhang Y, Deng M, Niu F, Yang B, Wang X, Wang B, Liang W, Deyholos MK, Yiang Y-Q (2014) Identification, expression and interaction analyses of calcium-dependent protein kinase (CPK) genes in canola (Brassica napus L.). BMC Genomics 15:211

Zhu JK (2003) Salt and drought stress signal transduction in plants. Annu Rev Plant Biol 53:247-273

Ziolkowski PA, Kaczmarek M, Babula D, Sadowski J (2006) Genome evolution in Arabidopsis/Brassica: conservation and divergence of ancient rearranged segments and their breakpoints. Plant $\mathbf{J}$ 47:67-74 